\title{
Different anterolateral procedures have variable impact on knee kinematics and stability when performed in combination with anterior cruciate ligament reconstruction
}

\author{
Thomas Neri @ (1, 1 2 Danè Dabirrahmani, ${ }^{3}$ Aaron Beach, ${ }^{1}$ Samuel Grasso, ${ }^{1}$ Sven Putnis, \\ Takeshi Oshima, ${ }^{1}$ Joseph Cadman, ${ }^{3}$ Brian Devitt, ${ }^{4}$ Myles Coolican, ${ }^{1}$ Brett Fritsch, \\ Richard Appleyard, ${ }^{3}$ David Parker ${ }^{1}$
}

${ }^{1}$ Sydney Orthopaedic Research Institute Ltd, Chatswood, New South Wales, Australia ${ }^{2}$ Laboratory of Human Movement Biology (LIBM EA 7424), University of Lyon-Jean Monnet, Saint Etienne, France ${ }^{3}$ Faculty of Medicine and Health Sciences, Macquarie University, Sydney, New South Wales, Australia

${ }^{4}$ OrthoSport Victoria, Richmond, Victoria, Australia

Correspondence to

Dr Thomas Neri, Sydney Orthopaedic Research Institute Ltd, Chatswood, NSW 2067, Australia;

thomas.neri@outlook.com

Finalist of the ISAKOS Albert Trillat Young Investigator Award 2019

Accepted 22 October 2020 Published Online First 24 November 2020

\section{Linked}

- http://dx.doi.org/10.1136/ jisakos-2020-000529

Check for updates

(C) International Society of Arthroscopy, Knee Surgery and Orthopaedic Sports Medicine 2021. No commercial re-use See rights and permissions. Published by BMJ.

\section{To cite: Neri T,}

Dabirrahmani D,

Beach $\mathrm{A}$, et al. J ISAKOS

2021:6:74-81.

\section{ABSTRACT}

Objective The optimal anterolateral procedure to control anterolateral rotational laxity of the knee is still unknown. The objective was to compare the ability of five anterolateral procedures performed in combination with anterior cruciate ligament reconstruction (ACLR) to restore native knee kinematics in the setting of a deficient anterior cruciate ligament $(A C L)$ and anterolateral structures.

Methods A controlled laboratory study was performed using 10 fresh-frozen cadaveric whole lower limbs with intact iliotibial band. Kinematics from $0^{\circ}$ to $90^{\circ}$ of flexion were recorded using a motion analysis three-dimensional (3D) optoelectronic system, allowing assessment of internal rotation (IR) and anteroposterior (AP) tibial translation at $30^{\circ}$ and $90^{\circ}$ of flexion. Joint centres and bony landmarks were calculated from 3D bone models obtained from CT scans. Intact knee kinematics were assessed initially, followed by sequential section of the $\mathrm{ACL}$ and anterolateral structures (anterolateral ligament, anterolateral capsule and Kaplan fibres). After ACLR, five anterolateral procedures were performed consecutively on the same knee: ALLR, modified Ellison, deep Lemaire, superficial Lemaire and modified Maclntosh. The last three procedures were randomised. For each procedure, the graft was fixed in neutral rotation at $30^{\circ}$ of flexion and with a tension of $20 \mathrm{~N}$.

Results Isolated ACLR did not restore normal overall knee kinematics in a combined ACL plus anterolateraldeficient knee, leaving a residual tibial rotational laxity $(p=0.034)$. Only the ALLR ( $p=0.661)$ and modified Ellison procedure $(p=0.641)$ restored overall IR kinematics to the normal intact state. Superficial and deep Lemaire and modified Maclntosh tenodeses overconstrained IR, leading to shifted and different kinematics compared with the intact condition $(p=0.004, p=0.001$ and $p=0.045$, respectively). Compared with ACLR state, addition of an anterolateral procedure did not induce any additional control on AP translation at $30^{\circ}$ and $90^{\circ}$ of flexion (all $p>0.05$ ), except for the superficial Lemaire procedure at $90^{\circ}(p=0.032)$.

Conclusion In biomechanical in vitro setting, a comparison of five anterolateral procedures revealed that addition of either ALLR or modified Ellison procedure restored overall native knee kinematics in a combined ACL plus anterolateral-deficient knee. Superficial and deep Lemaire and modified Maclntosh tenodeses achieved excellent rotational control but overconstrained $\mathrm{IR}$, leading to a change from intact knee kinematics.

\section{What are the new findings}

- Isolated ACLR failed to restore overall intact knee kinematics in a combined anterior cruciate ligament $(A C L)$ plus anterolateral- deficient knee, suggesting that unaddressed anterolateral injuries can lead to residual rotational laxity and that an anterolateral procedure may be necessary. This provides a rationale for undertaking an ACLR in combination with an anterolateral procedure in appropriate selected populations

- Addition of either ALLR or modified Ellison procedure restored overall native knee kinematics, suggesting that these additional procedures can be considered as a mechanical reinforcement to protect the $A C L$ graft during its integration. This would be potentially useful in primary $A C L$ reconstruction, helping to avoid re-injury without risking overconstraint.

- Superficial and deep Lemaire and modified Maclntosh tenodeses achieved excellent rotational control but overconstrained internal rotation, leading to non-physiological kinematics. This additional control may be useful in revision surgery.

Level of evidence The level-of-evidence statement does not apply for this laboratory experiments study.

\section{INTRODUCTION}

Despite a long history of differing surgical procedures, controlling anterolateral rotational laxity of the knee after anterior cruciate ligament (ACL) rupture remains a challenge. ${ }^{1}$ The results of a primary intra-articular anterior cruciate ligament reconstruction (ACLR) are excellent, with approximately $90 \%$ of patients achieving normal or nearnormal knee function. ${ }^{2}$ However, these results are not universal. Studies have reported 11\%-30\% recurrent and persistent instability. ${ }^{3-5}$ This can affect a patient's ability to return to high-demand labour or high-level sport, ${ }^{3}$ and is a cause of graft failure. ${ }^{6}$ To resolve this recurrent anterolateral rotational laxity, tunnel positions have changed, and anatomical double-bundle ACLR was developed to 
improve control of both anterior tibial translation and internal tibial rotation. However, these new techniques have not definitively demonstrated clinical superiority, ${ }^{7}$ suggesting that in some patients an intra-articular reconstruction is not sufficient to control anterolateral rotational laxity. ${ }^{8}$

A combined ACLR and anterolateral procedure has been suggested to improve control of anterolateral rotational laxity over an isolated ACLR, with several studies demonstrating a clinical advantage. ${ }^{9-11}$ Numerous anterolateral procedures are available, although they can essentially be divided into two broad groups: anterolateral ligament reconstruction (ALLR) and lateral extra-articular tenodesis (LET) using a section of iliotibial band (ITB). ${ }^{12}$

Methods of performing a LET were described by Lemaire in $1967,{ }^{13}$ MacIntosh in $1972,{ }^{14}$ and Ellison in $1979^{15}$ as novel ways of controlling anterolateral rotational laxity, and these eponymous terms are still used today. Further modifications introduced an intra-articular element by passing the ITB graft around the lateral femoral condyle and into the knee, becoming the first examples of a combined intra-articular reconstruction of the ACL alongside an anterolateral extra-articular reconstruction.

A Segond fracture, which is a bony avulsion of the anterolateral complex (ALC) from the tibia, is also indicative of ACL rupture. ${ }^{16}$ Recognition of this led to the concept of a purely soft tissue lateral extra-articular injury in association with an ACL rupture. Subsequent anatomical studies allowed us to better understand the function of these structures. ${ }^{16-18}$ Among them, the anterolateral ligament (ALL) emerged as a structure important in the understanding of anterolateral rotational laxity ${ }^{19} 20$ and has led to development and promotion of techniques describing reconstruction of this ligament. ${ }^{921}$

Determining which anterolateral procedure best controls anterolateral rotational laxity with the least detrimental effect on knee kinematics remains a challenge, with a lack of direct comparisons leaving the decision to the surgeon's own judgement and experience. The objective of this study was therefore to compare the ability of a variety of anterolateral procedures performed, in combination with ACLR, to restore native knee kinematics in the setting of a deficient ACL and anterolateral structures. It was hypothesised that LET would reduce more tibial internal rotation (IR) than an ALLR but would further overconstrain the knee.

\section{METHODS}

\section{Specimen preparation}

A controlled laboratory study was performed using 10 freshfrozen cadaveric knees from five half bodies (three men and two women). We used the whole leg and pelvis in order to keep the ITB intact, as well as all other synergistic biarticular structures crossing the hip and/or the knee. The specimens showed no signs of degeneration and were exposed to room temperature for 24 hours prior to the experimentation. The specimens were procured from a tissue bank after approval from the local research ethics committee.

Examination of the knee and an anteromedial knee arthroscopy were performed to check the ACL status. Exclusion criteria were specimens with an ACL injury (a positive anterior tibial drawer, a positive pivot shift test or an arthroscopic ACL tear), severe deformity, severe knee osteoarthritis and previous knee surgery.

\section{Experimental set-up}

The specimen was fixed on a bench to control both pelvic and the femoral movements, with the lower leg allowed to hang free in order to easily apply movement (knee extension to full flexion, internal and external rotation).

Each knee was prepared with four bicortical pins (two for the femur and two for the tibia) placed to avoid any soft tissue impingement. Two retroreflective sensors were fixed to each pin. Consequently, the femur and the tibia were equipped with four targets each: F1-F4 and T1-T4 (figure 1A). After pin insertion, a CT scan was performed to allow registration of the reference coordinate system using a standardised and previously validated imaging protocol. ${ }^{22}$ Joint centres and bone landmarks were calculated from three-dimensional (3D) bone models obtained as defined in the International Society of Biomechanics (ISB) conventions and by Grood and Suntay. ${ }^{23} 24$

Kinematics from $0^{\circ}$ to $90^{\circ}$ of knee flexion were recorded using a Motion Analysis 3D optoelectronic system (Vicon, Los Angeles, USA). The system consisted of five high-definition Vicon Bonita cameras on tripods operating at $100 \mathrm{~Hz}$ (figure 1B). After installation and calibration around the working area, the system followed the retroreflective sensors. The kinematics were interpolated to obtain values for each degree of knee flexion from $0^{\circ}$ to $90^{\circ}$. In a preliminary work, this tracking system has been reported to have a translational
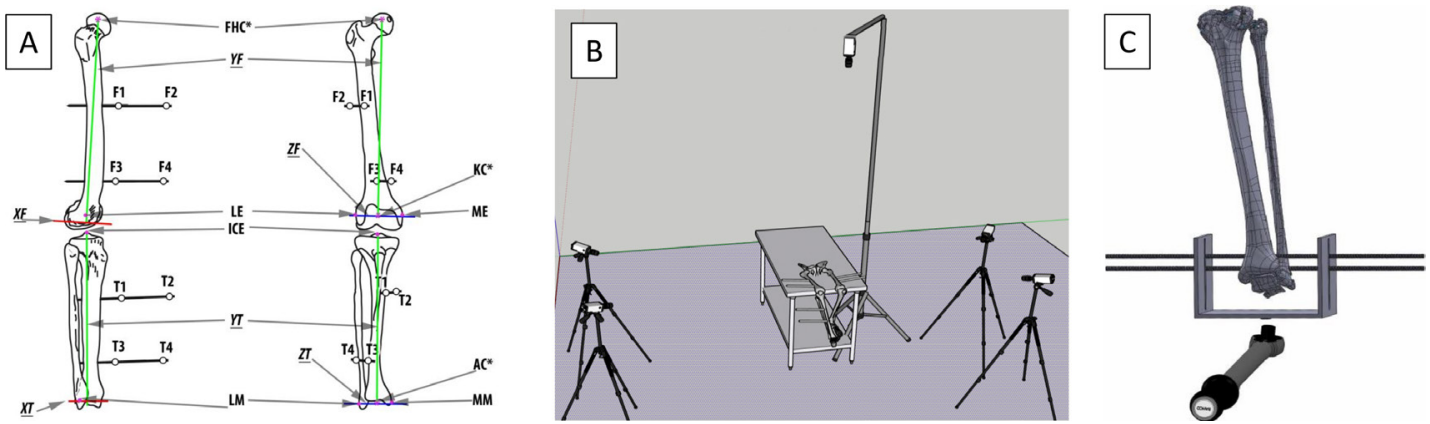

Figure 1 (A) Definition of bone landmarks, bone axis and joint centres. Femur: F1, F2, F3, F4, FHC * LE, ME, KC*. Tibia: T1, T2, T3, T4, centre of ICE. Ankle: MM, LM, AC* . Purple asterisks and circles indicate calculated landmarks and joint centres. Femoral axis: XF (in red), YF (in green), ZF (in blue). Tibial axis: $\mathrm{XT}$ (in red), YT (in green), ZT (in blue). (B) Installation of motion analysis system with five high-definition cameras, around the specimen. (C) Dynamometric torque rig used to control the tibial rotation applied. Ridged rig fixation was with two extra-articular Bmalleolar (distal tibia and fibula) pins. AC, ankle centre; FHC, femoral head centre; ICE, intercondylar eminence; KC, knee centre; LE, lateral epicondyle; LM, lateral malleolus; ME, medial epicondyle; MM, medial malleolus. 


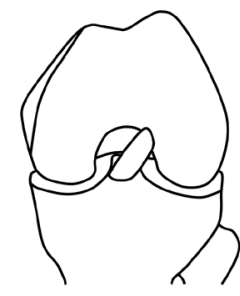

1-Intact knee

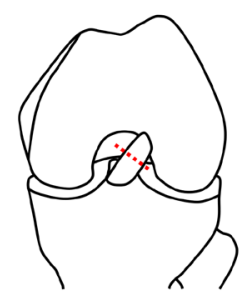

2 - ACL section

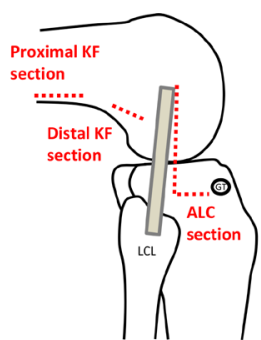

3 -combined ACL plus terolateral-deficient knee

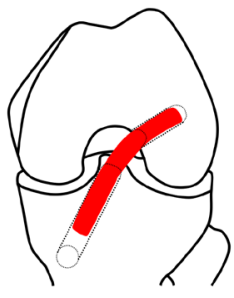

4- ACL reconstruction

】

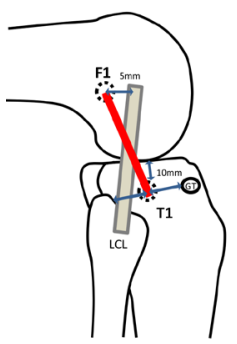

ALL reconstruction

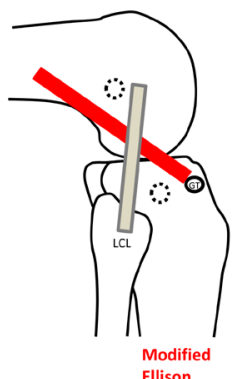

Mllison

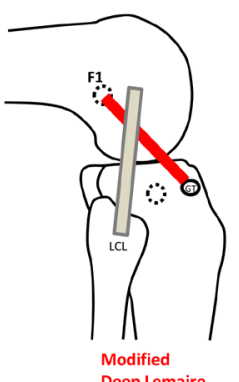

Deep Lemaire
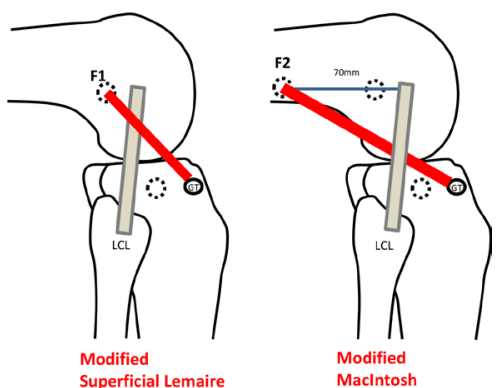

Figure 2 Kinematic data were recorded from the following nine consecutive knee conditions: (1) intact, (2) ACL deficient knee, (3) combined ACL plus anterolateral-deficient knee, (4) isolated ACLR, (5) combined ACLR and ALLR, (6) combined ACLR and modified Ellison procedure, (7) combined ACLR and modified deep Lemaire procedure, (8) combined ACLR and modified superficial Lemaire procedure, (9) combined ACLR and modified Maclntosh procedure. The last three procedures (deep and superficial Lemaire and modified Maclntosh) were randomised. ACL, anterior cruciate ligament; ALC, anterolateral complex; ALL, anterolateral ligament; ALLR, anterolateral ligament reconstruction; F1, femoral tunnel position for all reconstruction and Lemaire procedures ( $5 \mathrm{~mm}$ posterior and proximal to the femoral epicondyle); F2, femoral tunnel position for modified Maclntosh procedure (70 $\mathrm{mm}$ posterior and proximal to the femoral epicondyle); GT, Gerdy's tubercle; KF, Kaplan fibre; LCL, lateral collateral ligament; T1, tibial tunnel position for all reconstruction (equidistant from the centre of GT and the anterior margin of the fibular head and $10 \mathrm{~mm}$ distal to the joint line.

accuracy of less than $0.04 \mathrm{~mm}$ and a rotational accuracy of less than $0.2^{\circ} .25$

\section{Surgical techniques and conditions}

Surgery was performed by a single experienced orthopaedic surgeon in all specimens. Nine conditions were successively applied for each knee (figure 2). Following assessment of the normal intact knees (condition 1: intact), arthroscopic ACL transection was performed (condition 2: ACL deficient knee). An ALC transection was then achieved, including ALL, anterolateral capsule and proximal and distal Kaplan fibre sections, without damaging the ITB by retracting it anteriorly (condition 3: combined ACL plus anterolateral-deficient knee). ${ }^{18}$

After obtaining an ACL and ALC-deficient knee, an ACLR was performed with a quadrupled hamstring autograft (semitendinosus) fixed using adjustable cortical suspensory fixation in the femur and tibia (Graftlink system, Arthrex, Florida, USA) (condition 4: isolated ACLR).

After ACLR, 5 anterolateral procedures were performed on the same knee: ALLR, modified Ellison, deep Lemaire, superficial Lemaire and modified MacIntosh. To allow comparison between procedures, the graft was always fixed in the same condition: in neutral rotation at $30^{\circ}$ of flexion and with $20 \mathrm{~N}$ of applied tension (figure 3). ${ }^{2627}$ This degree of flexion was chosen because under in vivo conditions, the loaded knee requires rotational control in the early degrees of flexion. The same operator inserted each interference screw, ensuring a controlled speed without displacement of the graft each time, to minimise further graft tensioning.

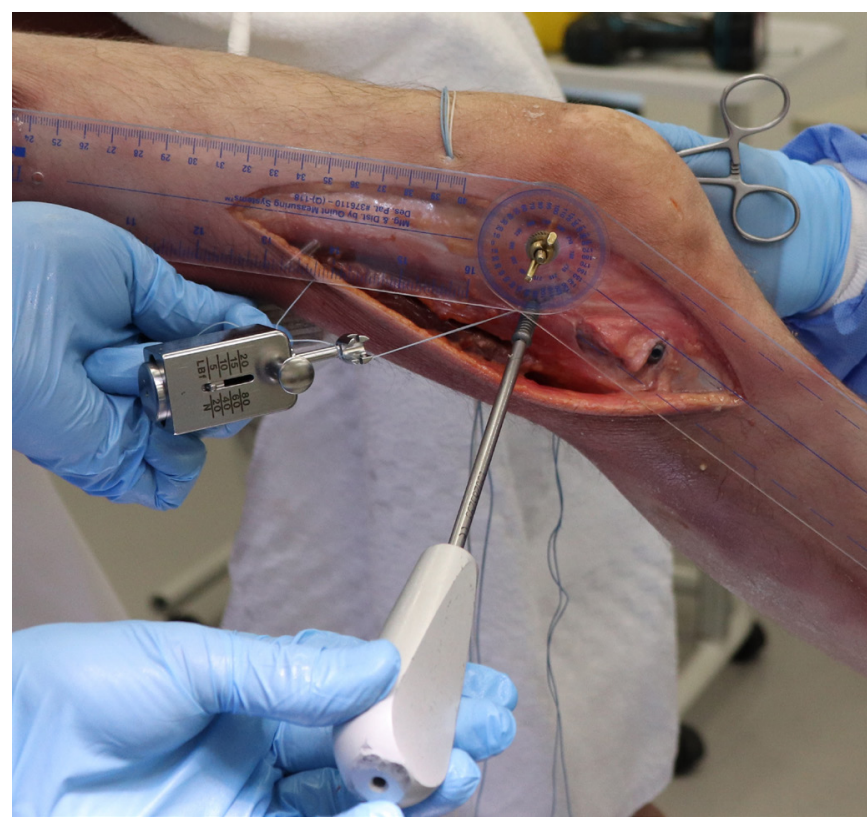

Figure 3 Lateral photography of femoral fixation for the superficial Lemaire procedure. In all the anterolateral procedures performed, the graft was always fixed in the same condition: in neutral rotation at $30^{\circ}$ of flexion and with $20 \mathrm{~N}$ of applied tension, controlled by a dynamometer through a pulling suture fixed to the graft at the entry of the femoral tunnel. 
- Condition 5: a combined ACLR and ALLR was performed with a free gracilis graft passing under the ITB and over the lateral collateral ligament (LCL), according to the Chahla technique. ${ }^{21}$ The graft was passed into closed-socket tunnels and fixed with interference screws. The tibial tunnel was located equidistant from the centre of Gerdy's tubercle (GT) and the anterior margin of the fibular head and $10 \mathrm{~mm}$ distal to the joint line. ${ }^{18}{ }^{28}$ The femoral socket was located $5 \mathrm{~mm}$ proximal and posterior to the LCL's femoral insertion. 182829

- Condition 6: a combined ACLR and modified Ellison procedure modified by Devitt et al was performed. ${ }^{15}{ }^{30}$ It consisted of using a $15 \mathrm{~mm}$-wide central strip of ITB detached distally by performing a thin GT osteotomy. This strip was passed underneath the LCL and the bone fixed back to the GT bed. Fixation was with $3.5 \mathrm{~mm}$ suture anchors reinforced with a staple. The ITB defect was then closed.

- Condition 7: a combined ACLR and modified deep Lemaire procedure was performed using a $15 \times 100 \mathrm{~mm}$ central strip of the ITB passed under the LCL and fixed in the same tunnel as the ALLR with an interference screw.

- Condition 8: a combined ACLR and modified superficial Lemaire was performed using the same graft and the same femoral fixation site as condition 7 , but with the graft positioned over the LCL.

- Condition 9: a combined ACLR and modified MacIntosh was performed by using a $15 \times 150 \mathrm{~mm}$ central strip of ITB, passed underneath the LCL and through a further closedsocket tunnel $70 \mathrm{~mm}$ proximal to the femoral epicondyle at the insertion of the lateral intermuscular septum and fixed with an interference screw. ${ }^{27}$

\section{Kinematics analysis and testing protocol}

Kinematic data were recorded from the nine consecutive knee conditions. For reasons of ITB preservation and feasibility, only the last three procedures were randomised to avoid any potential bias due to deterioration of the tissue. The sequence of conditions tested was dictated by the anatomical requirements of the procedures. The ALLR must be tested first before using the ITB. Ellison procedure was to be the second condition tested because the graft had to be intact in the proximal portion.

Knee kinematics were recorded from three cycles through $0^{\circ}$ to $90^{\circ}$ of passive knee flexion obtained by hand, with IR applied. For all knee kinematics, a simultaneous IR torque was applied to the leg. IR was standardised with torque applied to the tibia, provided by a dynamometric torque rig placed at the tibiofibular mortise, triggering at $5 \mathrm{Nm}$ (figure 1C). Zero degree of flexion was defined as the position at which the tibial and femoral rods were parallel, as seen in the sagittal plane. After processing the kinematics using Visual3D software, the data were filtered (Butterworth filter of order 4 with a cut-off frequency of $6 \mathrm{~Hz}$ ) according to Winter et al and Pezzack et al. ${ }^{3132}$ The kinematics were interpolated to obtain values for each degree of knee flexion from $0^{\circ}$ to $90^{\circ}$.

Anteroposterior (AP) translation was tested by inducing anterior tibial drawer, at $30^{\circ}$ (AP30) and $90^{\circ}$ (AP90) of knee flexion, under the control of a dynamometer set at $90 \mathrm{~N}$. AP translation was calculated as the perpendicular distance from the midpoint of the femoral epicondylar axis to the tibial coronal reference plane, and motions described were tibial movements relative to the femur.

The motion analysis system coupled with 3D CT modelling allows the position of the lower limb, including the knee, in a defined 3D space to be known at any time. During the kinematic assessment, the varus/valgus, flexion/extension, rotation and $\mathrm{AP}$ translation were known and therefore controlled.

\section{Statistical analysis}

All statistical analyses were performed using SPSS software, with a significance level set at $\mathrm{p}<0.05$.

A power calculation, based on a study using optical tracking system, ${ }^{27}$ determined that a sample size of 8 would allow the identification of changes in translation and rotation of $2.1 \mathrm{~mm}$ and $1.2^{\circ}$, respectively, with $80 \%$ power and $95 \%$ confidence. As there is the greatest SD of laxity, 10 knees were used to identify potentially small differences.

All kinematic data were subsequently analysed using a two-factor repeated measures analysis of variance with Bonferroni corrections. The two factors assessed were the conditions of the knee and the flexion angle of the knee. Comparisons were made using a contrast test, followed by tests for intrasubject correlations such as sphericity, Greenhouse-Geisser and Huynh-Feldt. Overall kinematics (through the full range of flexion: $0^{\circ}-90^{\circ}$ of flexion) relating to each condition was compared with the intact knee.

\section{RESULTS}

\section{Internal tibial rotation}

Kinematic outcomes $\left(0^{\circ}-90^{\circ}\right)$ for IR condition are reported in table 1 and figure 4 . IR laxity is induced by ACL transection $(\mathrm{p}<0.001)$ and amplified by ALC section $(\mathrm{p}<0.001)$. Isolated ACLR did not restore overall normal knee kinematics in the combined ACL and anterolateral-deficient knee, leaving a residual rotational laxity $(\mathrm{p}=0.034)$.

Only the ALLR and modified Ellison procedure restored overall IR kinematics, through the full range of flexion, to the normal intact state (no statistical difference with intact condition: $\mathrm{p}=0.661$ and $\mathrm{p}=0.641$, respectively). From $0^{\circ}$ to $90^{\circ}$ of flexion, the addition of ALLR induced a significant additional control compared with isolated ACLR, allowing a return to normal overall kinematics $(p=0.661)$. The additional modified Ellison procedure induced a significant IR decrease during

\begin{tabular}{|c|c|c|c|c|c|c|c|c|c|}
\hline & Intact & ACL section & $\begin{array}{l}\text { Combined ACL-anterolateral- } \\
\text { deficient knee }\end{array}$ & ACLR & ACLR+ALLR & ACLR+Ellison & $\begin{array}{l}\text { ACLR+superficial } \\
\text { Lemaire }\end{array}$ & $\begin{array}{l}\text { ACLR+deep } \\
\text { Lemaire }\end{array}$ & ACLR+Maclntosh \\
\hline $0^{\circ}$ & $0 \pm 0$ & $2.55 \pm 1.41$ & $4.36 \pm 1.35$ & $2.05 \pm 1.96$ & $0.43 \pm 1.04$ & $0.38 \pm 1.75$ & $1.27 \pm 1.63$ & $-1.86 \pm 1.96$ & $-0.97 \pm 2.35$ \\
\hline $15^{\circ}$ & $7.5 \pm 0.52$ & $9.73 \pm 1.29$ & $11.39 \pm 1.75$ & $8.78 \pm 2.36$ & $7.4 \pm 2.11$ & $5.84 \pm 2.08$ & $5.83 \pm 2.49$ & $2.58 \pm 2.24$ & $3.98 \pm 2.43$ \\
\hline $30^{\circ}$ & $10.59 \pm 1.18$ & $12.38 \pm 1.1$ & $14.22 \pm 1.78$ & $11.64 \pm 1.49$ & $10.22 \pm 0.89$ & $9.16 \pm 1.82$ & $7.35 \pm 2.12$ & $5.39 \pm 2.12$ & $6.83 \pm 2.28$ \\
\hline $45^{\circ}$ & $10.35 \pm 1.29$ & $12.11 \pm 2.08$ & $13.55 \pm 2.13$ & $11.64 \pm 1.75$ & $10.85 \pm 1.36$ & $10.85 \pm 1.69$ & $7.17 \pm 2.08$ & $6.79 \pm 1.49$ & $8.22 \pm 2.1$ \\
\hline $60^{\circ}$ & $9.76 \pm 1.26$ & $11.63 \pm 2.16$ & $12.75 \pm 1.95$ & $10.76 \pm 2.32$ & $10.23 \pm 2.08$ & $11.36 \pm 2.32$ & $6.49 \pm 2.29$ & $7.04 \pm 1.75$ & $8.04 \pm 2.41$ \\
\hline $75^{\circ}$ & $9.96 \pm 2.01$ & $11.7 \pm 2.46$ & $12.64 \pm 2.11$ & $10.41 \pm 2.37$ & $10.06 \pm 2.26$ & $11.82 \pm 2.36$ & $5.88 \pm 2.36$ & $8.02 \pm 2.32$ & $8.57 \pm 2.37$ \\
\hline $90^{\circ}$ & $11.49 \pm 2.26$ & $13.06 \pm 2.48$ & $13.72 \pm 2.1$ & $11.88 \pm 1.96$ & $11.53 \pm 2.29$ & $13.06 \pm 2.48$ & $5.85 \pm 2.36$ & $9.48 \pm 2.67$ & $9.77 \pm 2.27$ \\
\hline
\end{tabular}

$\mathrm{ACL}$, anterior cruciate ligament; $\mathrm{ACLR}$, anterior cruciate ligament reconstruction; $\mathrm{ALLR}$, anterolateral ligament reconstruction. 


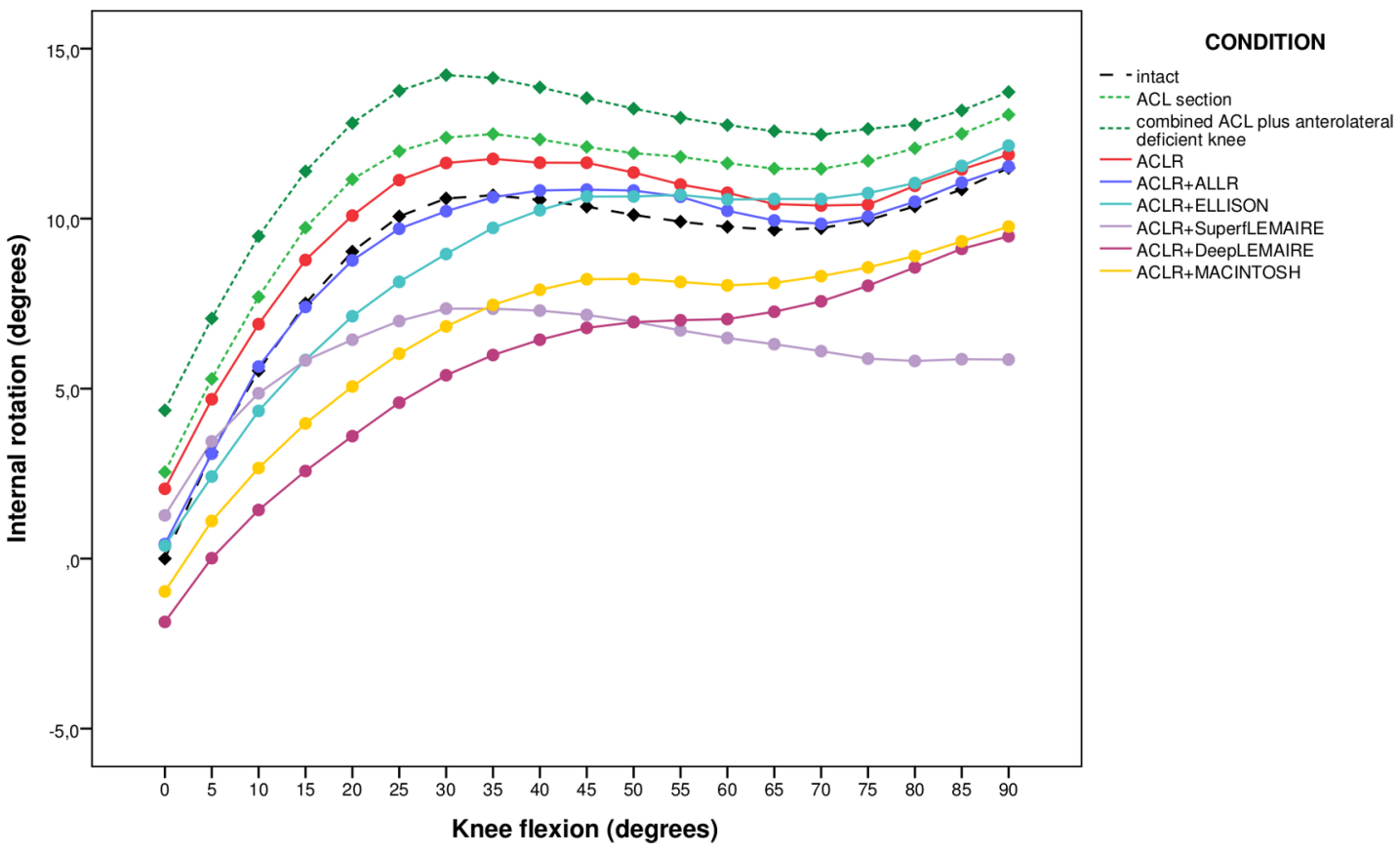

Figure 4 Kinematics comparison from $0^{\circ}$ to $90^{\circ}$ of flexion, when the knee is internally rotated between the different knee conditions. Comprehensive data and SD are provided in table 1. ACL, anterior cruciate ligament; $A C L R$, anterior cruciate ligament reconstruction; ALLR, anterolateral ligament reconstruction.

$0^{\circ}-45^{\circ}$ flexion $(\mathrm{p}=0.010)$ with a lack of rotational control beyond this range $(p=0.114)$. Regardless of the degree of knee flexion, superficial and deep Lemaire and modified MacIntosh tenodeses are more efficient at IR control. They overconstrained IR, leading to shifted and different kinematics compared with the intact condition $(p=0.004, p=0.001$ and $p=0.045$, respectively). Visual comparison between superficial Lemaire and deep Lemaire/modified MacIntosh kinematics curves, suggested that passing the ITB over the LCL induced an additional overconstraint from $45^{\circ}$ to $90^{\circ}$ of flexion.

\section{Anterior tibial translation}

Increased AP laxity at $30^{\circ}$ and $90^{\circ}$ of knee flexion is induced by ACL transection $(\mathrm{p}<0.001)$ and amplified by ALC section $(p=0.01)$ (table 2 and figure 5). Compared with the intact knee, isolated ACLR corrected the AP translation at $90^{\circ}$ of knee flexion (no statistical difference with intact condition, $\mathrm{p}=0.064$ ) but not completely when at $30^{\circ}$ of flexion $(p=0.016)$.

At $30^{\circ}$ of flexion, addition of an anterolateral procedure did not induce any additional control on AP translation compared with the isolated ACLR condition (all $\mathrm{p}>0.05$ ) and also did not restore the intact condition (all $\mathrm{p} \leq 0.01$ ). At $90^{\circ}$ of flexion, only the superficial Lemaire procedure induced an additional significant AP decrease compared with an isolated ACLR $(p=0.032)$.

\section{DISCUSSION}

This study is the first to compare the five main anterolateral procedures that are in clinical use today: ALLR, superficial and deep Lemaire, modified Ellison and modified MacIntosh procedures. Furthermore, it has a distinct advantage when compared with previous biomechanical studies by preserving the whole leg and pelvis, hence keeping the ITB and its synergistic biarticular function completely intact, and in doing so recreating a more clinically relevant scenario. Isolated ACLR failed to restore intact knee kinematics in a combined ACL plus anterolateral-deficient knee, suggesting that unaddressed anterolateral injuries can lead to residual rotational laxity and that an anterolateral procedure may be necessary. Among them, only addition of either ALLR or the modified Ellison procedure restored native knee kinematics. Superficial and deep Lemaire,and modified MacIntosh tenodeses provided additional control of IR but overconstrained knee kinematics.

Failure of isolated ACLR to restore intact knee kinematics in a combined ACL plus anterolateral-deficient knee could explain why $11 \%-30 \%$ of patients still have recurrent and persistent instability with a pivot shift test positive after an ACLR, ${ }^{3-5}$ and some of them remain dissatisfied. ${ }^{33} 34$ It suggests that unaddressed anterolateral injuries can lead to residual rotational laxity. ${ }^{35}$ With a reinjury rate of $6.5 \%-26.7 \%$ after an isolated ACLR, studies have sought to determine if this is reduced with an additional anterolateral procedure. ${ }^{3637}$ Rezende et al, through a recent clinical meta-analysis, demonstrated that the addition of a LET to an ACLR decreases persistent instability but had a nonsignificant tendency to reduce graft rupture rate. ${ }^{10}$ In a prospective and comparative study enrolling 618 patients, Getgood et al demonstrated that addition of LET to ACLR resulted to a reduction in graft rupture and persistent rotatory laxity at 2 years after surgery. ${ }^{11}$ For an ALLR, Thaunat et al (548 patients, mean follow-up of 35.5 months) reported a low rerupture rate of $2.6 \%$ after a combined ALLR and ACLR procedure. ${ }^{938}$

We have shown that there are significant kinematic differences between ALLR, modified Ellison and LET procedures. In agreement with our results, Spencer et al similarly reported that the ALLR would allow only minimal control of IR, whereas the LET would allow control of both rotation and translation. ${ }^{39}$ However, this work studied the effect of an anterolateral procedure on a combined ACL plus anterolateral-deficient knee without subsequent ACLR, which is difficult to interpret. Inderhaug et al demonstrated that only the modified MacIntosh and deep Lemaire procedures restored native knee kinematics. ${ }^{27}$ Here the effect of the ALLR was potentially underestimated because fixation was provided with anchors, while Lemaire 
Table 2 Anteroposterior translations data (mean \pm SD, in $\mathrm{mm}$ ) at $30^{\circ}$ and $90^{\circ}$ of flexion, for the nine knee conditions tested

\begin{tabular}{|c|c|c|c|c|c|c|c|c|c|}
\hline & Intact & ACL section & $\begin{array}{l}\text { Combined ACL-anterolateral- } \\
\text { deficient knee }\end{array}$ & ACLR & $A C L R+A L L R$ & ACLR+Ellison & $\begin{array}{l}\text { ACLR+superficial } \\
\text { Lemaire }\end{array}$ & ACLR+deep Lemaire & ACLR+MacIntosh \\
\hline $30^{\circ}$ & $5.83 \pm 1.79$ & $11.98 \pm 1.99$ & $13.1 \pm 2.39$ & $8.07 \pm 1.91$ & $8.34 \pm 1.44$ & $8.51 \pm 1.64$ & $8.04 \pm 1.48$ & $8.26 \pm 1.65$ & $8.26 \pm 1.76$ \\
\hline $90^{\circ}$ & $2.68 \pm 0.9$ & $5.5 \pm 1.57$ & $5.96 \pm 1.83$ & $3.36 \pm 1.44$ & $3.26 \pm 1.25$ & $3.39 \pm 1.46$ & $2.63 \pm 0.93$ & $2.88 \pm 0.93$ & $3.26 \pm 1.17$ \\
\hline
\end{tabular}

and modified MacIntosh procedures were fixed with interference screws. Another limitation was that this study was with the knee in its isolated state, therefore eliminating the biarticular effect of ITB and femoral biceps. Geeslin et al, in a robotic study, reported similar results to our study. ${ }^{26}$ They demonstrated that ALLR and LET procedures resulted in significant reductions of tibial IR versus the intact state independent of graft tension or fixation angle. However, this work analysed only deep Lemaire and ALLR on isolated knees.

It was to be expected that we would see biomechanical differences between each anterolateral procedure and this proved correct. The effect of knee flexion angle at the time of fixation does influence subsequent knee kinematics, ${ }^{26} 2740$ but this was not a factor in our study as it was controlled at $30^{\circ}$ of flexion in neutral rotation for all conditions. Differences in graft mechanical property are another proposed factor with a free gracilis graft for the ALLR and the ITB for the LET, but a recent study by Wytrykowski et al analysed the mechanical characteristics of the different grafts that can be used for ALLR. ${ }^{41}$ They found gracilis had a maximum load to failure of $200 \mathrm{~N}$, a stiffness of $131.7 \mathrm{~N} / \mathrm{mm}$ and an elongation at failure of $19 \mathrm{~mm}$, whereas ITB had a maximum load to failure of $160 \mathrm{~N}$, a stiffness of $39.9 \mathrm{~N} / \mathrm{mm}$ and an elongation at failure of $20.8 \mathrm{~mm}$. These small differences are not enough to explain the change in kinematics.

A more convincing argument was first introduced by $\mathrm{Amis}^{42}$ and then recently confirmed in a biomechanical study, ${ }^{40}$ and concerns the difference in geometry between anatomical fixation points of the grafts. While there is no difference in the femoral insertion point between the two techniques (they both use a tunnel positioned posterior and proximal to the femoral epicondyle), there is a difference in their tibial attachment sites. The tibial positioning of the LET is located at the level of the GT, which is therefore anterior (approximately $18-20 \mathrm{~mm}$ ) from that of the ALLR. Thus, the strength of resistance to IR of the tibia (in particular its AP horizontal component) will be greater for the LET than for the ALLR (figure 6).
Regarding the effect of passing the ITB graft over or under the LCL, our kinematic comparison demonstrated that LET with a graft passed over the LCL (superficial Lemaire) has more overconstraint in full flexion than LET with a graft passed under the LCL (deep Lemaire or modified MacIntosh). Biomechanically, there is no clear explanation. One hypothesis could be that in flexion the superficial graft has to travel over the epicondyle, which is a longer distance in flexion than a path under the LCL, which is closer to the bone. Conversely, passing the graft under the LCL creates a pulley effect, mainly in extension. Therefore, the deep graft would be expected to probably be tighter in extension and the superficial graft tighter in flexion. Despite no significant reported data, examination of figure 4 confirms this hypothesis. The superficial Lemaire has a tendency to be tighter in full flexion compared with the deep Lemaire and the modified MacIntosh, which are tighter close to the extension.

The modified Ellison technique restored intact kinematics without over constraining the knee. The explanation is probably that the lack of femoral attachment increases the range of variation of the graft length, making this procedure a less rigid system.

Some studies have investigated the relationship between the anterolateral procedures and the pivot-shift (PS) test. Getgood et al in a large randomised clinical trial compared ACLR with or without LET. In the ACLR group, $40 \%$ of patients sustained the primary outcome of isolated ACLR clinical failure (ie, persistent PS $\geq 1$ ), compared with $25 \%$ of ACLR+LET patients. ${ }^{11}$ For ALLR, Sonnery-Cottet et al reported less residual PS in the ALL group (9.1\%) compared with isolated ACLR (35.5\%). ${ }^{43}$ A recent meta-analysis comparing LET and ALLR showed that the proportion of knees that belonged to grade 2 or 3 on the PS test, indicating poor rotational stability, was similar between these two techniques. ${ }^{44}$

This study demonstrated that ALLR and modified Ellison procedures restored native knee kinematics at time 0 and that Lemaire (deep and superficial) and modified MacIntosh procedures achieved additional rotational control, but overconstrained
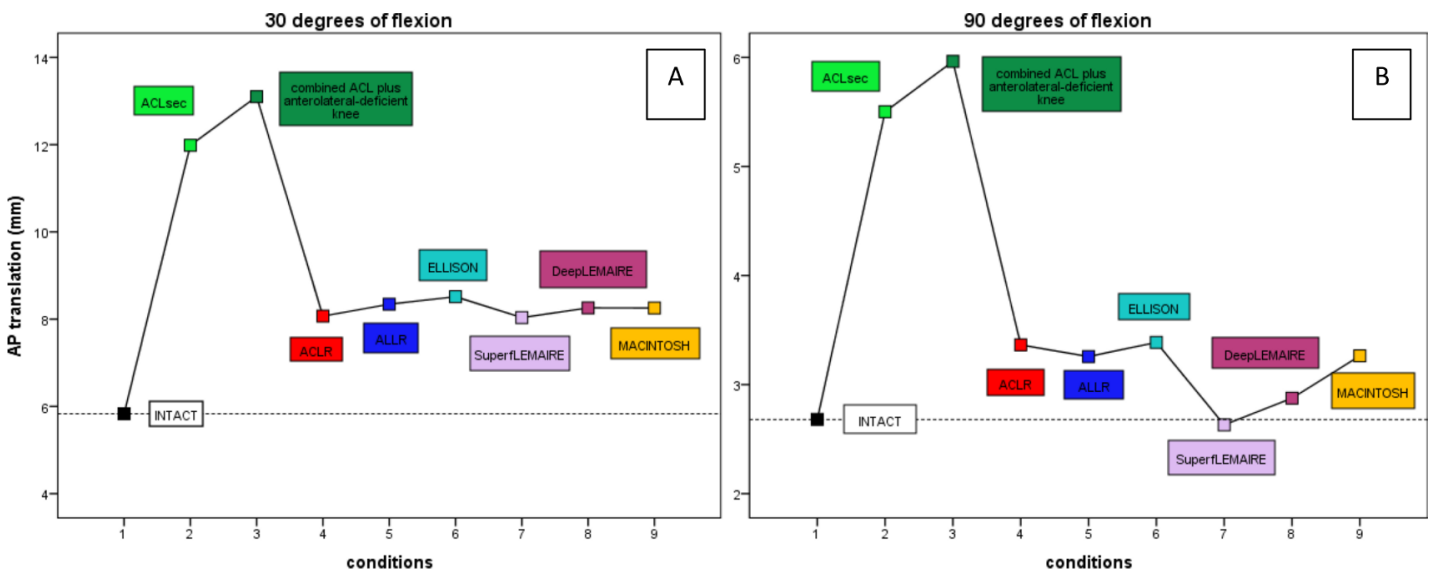

Figure $5 \mathrm{AP}$ translation comparison at $30^{\circ}$ and $90^{\circ}$ of flexion, between the nine studied conditions. Comprehensive data and SDs are provided in table 2. $A C L$, anterior cruciate ligament; $A C L R$, anterior cruciate ligament reconstruction; $A L L R$, anterolateral ligament reconstruction; $A P$, anteroposterior. 


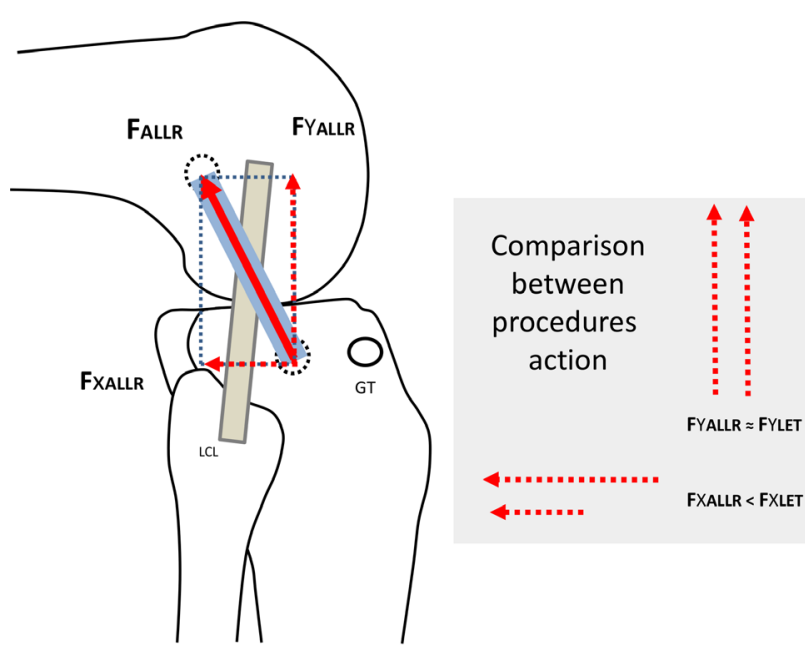

ALLR

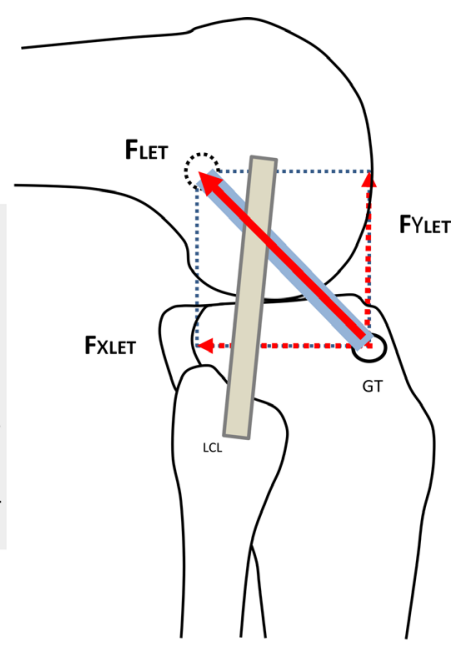

LET: Modified Lemaire

Figure 6 Biomechanical considerations for graft location explaining kinematic differences between ALLR and modified Lemaire LET. The FxLET is higher than the FXALL. FALLR is the overall action of ALLR, with horizontal (FXALLR) and vertical (FYALLR) components. FLET is the overall action of LET, with horizontal (FxLET) and vertical (FYLET) components). ALL, anterolateral ligament; ALLR, anterolateral ligament reconstruction; FxALL, horizontal component of the anterolateral ligament; FxLET, horizontal component of the lateral extra-articular tenodenis; LET, lateral extra-articular tenodenis.

the knee by decreasing the IR. Based on these results, we could possibly suggest different indications for the different procedures. In primary ACLR with combined ACL plus anterolateraldeficient knee, the main goal is to restore intact kinematics and to protect the graft during its integration. Although there is no clear consensus on the indication for adding extra-articular reconstruction to an ACLR, some authors advocate their use in cases of combined ACL and anterolateral structure injuries (ALL injury confirmed by MRI or ultrasound, Segond fracture) and/or high-risk patients. ${ }^{45}$ For these injuries/patients, this study suggests that if required, the addition of either the ALLR or modified Ellison procedure could provide additional rotational control, protecting the ACL graft without risking overconstraint. In revision surgery, particularly with more chronic, gross laxity, where the priority is to avoid further graft rupture in a high-risk patient, the additional rotation control provided by modified MacIntosh and Lemaire tenodesis may be preferred.

There were limitations associated with this study. As with every biomechanical study, it is a 'time 0' assessment, which does not evaluate the effects of soft tissue healing or attenuation. The relevance is applicable only to the direct postoperative biomechanical condition and limited relevance to the in-vivo condition where the tissue starts to remodel and adapt over the course of the postoperative rehabilitation. While we randomised the last three procedures, the Lemaire and MacIntosh procedures were systematically performed after the Ellison procedure. By repeated testing of the ITB strip, this may affect the result of the following test conditions by creating either a stiffer or weaker reconstruction. Although all the biarticular structures were conserved, the study only measured the static effects of knee stabilisation, with no consideration of the active effects of dynamic stabilisers, such as the femoral biceps or the ITB. The simulated anterolateral injuries performed represent a worst case scenario, and while combined ACL and anterolateral injuries exist, they do not correspond to all ACL-deficient knees. There is likely a variability related to the mechanism and the magnitude of these combined injuries, and therefore, this study cannot be applied to a general ACL-injured population. One of the next challenges is therefore to identify patients with ACL and anterolateral injuries who would be appropriate candidates for a combined reconstruction.

\section{CONCLUSION}

In a biomechanical in vitro setting, isolated ACLR failed to restore overall intact knee kinematics in a combined ACL plus anterolateral-deficient knee, providing a rationale for undertaking an ACLR in combination with an anterolateral procedure in appropriately selected populations. A comparison of a variety of currently used anterolateral procedures revealed that addition of either ALLR or modified Ellison procedure restored overall native knee kinematics in a combined ACL plus anterolateraldeficient knee. While, superficial and deep Lemaire and modified MacIntosh tenodeses achieved excellent rotational control, we found these procedures added further constraint to IR, leading to a change from intact knee kinematics.

Contributors All authors were fully involved in the study. They have read and approved the manuscript. TN: conception, acquisition, analysis, data interpretation, work drafting, final approval, final agreement. DD: analysis, work drafting, final approval, final agreement. AB: conception, acquisition, analysis, work drafting, final approval, final agreement. SG: conception, analysis, data interpretation, work drafting, final approval, final agreement. SP: acquisition, data interpretation, work drafting, final approval, final agreement. TO: acquisition, work drafting, final approval, final agreement. JC: analysis, work drafting, final approval, final agreement. $\mathrm{BD}$ : conception, work drafting, final approval, final agreement. MC: conception, work drafting, final approval, final agreement. BF: conception, work drafting, final approval, final agreement. RA: conception, analysis, work drafting, final approval, final agreement. DP: conception, analysis, data interpretation, work drafting, final approval, final agreement.

Funding The authors have not declared a specific grant for this research from any funding agency in the public, commercial or not-for-profit sectors.

Competing interests None declared.

Patient consent for publication Not required.

Ethics approval This study obtained ethics approval from Surgical Skills and Anatomy Centre, Faculty of Medicine and Science, Macquarie University (application for approval to undertake research involving cadavers). 
Provenance and peer review Not commissioned; externally peer reviewed.

Data availability statement Data are available upon reasonable request.

\section{ORCID iD}

Thomas Neri http://orcid.org/0000-0001-8793-1956

\section{REFERENCES}

1 Guenther D, Griffith C, Lesniak B, et al. Anterolateral rotatory instability of the knee. Knee Surg Sports Traumatol Arthrosc 2015;23:2909-17.

2 Ardern CL, Webster KE, Taylor NF, et al. Return to sport following anterior cruciate ligament reconstruction surgery: a systematic review and meta-analysis of the state of play. Br I Sports Med 2011;45:596-606.

3 Kocher MS, Steadman JR, Briggs K, et al. Determinants of patient satisfaction with outcome after anterior cruciate ligament reconstruction. J Bone Joint Surg Am 2002;84:1560-72.

4 Anderson AF, Snyder RB, Lipscomb AB. Anterior cruciate ligament reconstruction. A prospective randomized study of three surgical methods. Am J Sports Med 2001:29:272-9.

5 Eriksson E. How good are the results of ACL reconstruction? Knee Surg Sports Traumatol Arthrosc 1997;5:137.

6 Kamath GV, Redfern JC, Greis PE, et al. Revision anterior cruciate ligament reconstruction. Am J Sports Med 2011;39:199-217.

7 Yasuda K, Tanabe Y, Kondo E, et al. Anatomic double-bundle anterior cruciate ligament reconstruction. Arthroscopy 2010;26:521-34.

8 Tashman S, Collon D, Anderson K, et al. Abnormal rotational knee motion during running after anterior cruciate ligament reconstruction. Am J Sports Med 2004:32:975-83.

9 Sonnery-Cottet B, Thaunat M, Freychet B, et al. Outcome of a combined anterior cruciate ligament and anterolateral ligament reconstruction technique with a minimum 2-year follow-up. Am J Sports Med 2015;43:1598-605.

10 Rezende FC, de Moraes VY, Martimbianco ALC, et al. Does combined intra- and Extraarticular ACL reconstruction improve function and stability? A meta-analysis. Clin Orthop Relat Res 2015;473:2609-18.

11 Getgood AMJ, Bryant DM, Litchfield R, et al. Lateral extra-articular Tenodesis reduces failure of hamstring tendon autograft anterior cruciate ligament reconstruction: 2 -year outcomes from the stability study randomized clinical trial. Am J Sports Med 2020;48:285-97.

12 DePhillipo NN, Cinque ME, Chahla J, et al. Anterolateral ligament reconstruction techniques, biomechanics, and clinical outcomes: a systematic review. Arthroscopy 2017;33:1575-83.

13 Lemaire M. Ruptures anciennes Du ligament croise anterieur Du genou. J Chir 1967:93:311-20.

14 Barrett DS, Mackenney RP. Maclntosh-Jones reconstruction for the unstable knee. Injury 1991;22:282-6.

15 Ellison AE. Distal iliotibial-band transfer for anterolateral rotatory instability of the knee. J Bone Joint Surg Am 1979;61:330-7.

16 Claes S, Luyckx T, Vereecke $E$, et al. The Segond fracture: a bony injury of the anterolateral ligament of the knee. Arthroscopy 2014;30:1475-82.

17 Getgood A, Brown C, Lording T, et al. The anterolateral complex of the knee: results from the International alc consensus group meeting. Knee Surg Sports Traumatol Arthrosc 2019;27:166-76.

18 Neri T, Palpacuer F, Testa R, et al. The anterolateral ligament: anatomic implications for its reconstruction. Knee 2017;24:1083-9.

19 Neri T, Parker DA, Beach A, et al. Anterolateral ligament of the knee: what we know about its anatomy, histology, biomechanical properties and function. Surg Technol Int 2018:33:312-8.

20 Claes S, Vereecke E, Maes M, et al. Anatomy of the anterolateral ligament of the knee. J Anat 2013:223:321-8.

21 Chahla J, Menge TJ, Mitchell JJ, et al. Anterolateral ligament reconstruction technique: an Anatomic-Based approach. Arthrosc Tech 2016;5:e453-7.

22 Grasso S, Linklater J, Li Q, et al. Validation of an MRI protocol for routine quantitative assessment of tunnel position in anterior cruciate ligament reconstruction. Am J Sports Med 2018;46:1624-31.

23 Grood ES, Suntay WJ. A joint coordinate system for the clinical description of threedimensional motions: application to the knee. J Biomech Eng 1983;105:136-44.
24 Wu G, Siegler S, Allard P, et al. ISB recommendation on definitions of joint coordinate system of various joints for the reporting of human joint motion--part l: ankle, hip, and spine. International Society of Biomechanics. J Biomech 2002;35:543-8.

25 Neri T, Testa R, Laurendon L, et al. Determining the change in length of the anterolateral ligament during knee motion: a three-dimensional optoelectronic analysis. Clin Biomech 2019;62:86-92.

26 Geeslin AG, Moatshe G, Chahla J, et al. Anterolateral knee extra-articular stabilizers: a robotic study comparing anterolateral ligament reconstruction and modified Lemaire lateral extra-articular Tenodesis. Am J Sports Med 2018;46:607-16.

27 Inderhaug E, Stephen JM, Williams A, et al. Biomechanical comparison of anterolateral procedures combined with anterior cruciate ligament reconstruction. Am I Sports Med 2017:45:347-54.

28 Kennedy MI, Claes S, Fuso FAF, et al. The anterolateral ligament: an anatomic radiographic, and biomechanical analysis. Am J Sports Med 2015;43:1606-15.

29 Daggett M, Ockuly AC, Cullen M, et al. Femoral Origin of the Anterolateral Ligament: An Anatomic Analysis. Arthroscopy 2016;32:835-41.

30 Devitt BM, Lord BR, Williams A, et al. Biomechanical assessment of a distally fixed lateral extra-articular augmentation procedure in the treatment of anterolateral rotational laxity of the knee. Am J Sports Med 2019;47:2102-9.

31 Pezzack JC, Norman RW, Winter DA. An assessment of derivative determining techniques used for motion analysis. J Biomech 1977;10:377-82.

32 Winter DA, Sidwall HG, Hobson DA. Measurement and reduction of noise in kinematics of locomotion. J Biomech 1974;7:157-9.

33 Kamien PM, Hydrick JM, Replogle WH, et al. Age, graft size, and Tegner activity level as predictors of failure in anterior cruciate ligament reconstruction with hamstring autograft. Am J Sports Med 2013:41:1808-12.

34 Lind M, Menhert F, Pedersen AB. Incidence and outcome after revision anterior cruciate ligament reconstruction: results from the Danish Registry for knee ligament reconstructions. Am J Sports Med 2012;40:1551-7.

35 Samuelson M, Draganich LF, Zhou X, et al. The effects of knee reconstruction on combined anterior cruciate ligament and anterolateral capsular deficiencies. Am J Sports Med 1996;24:492-7.

36 Hettrich CM, Dunn WR, Reinke EK, et al. The rate of subsequent surgery and predictors after anterior cruciate ligament reconstruction: two- and 6-year follow-up results from a multicenter cohort. Am J Sports Med 2013:41:1534-40.

37 Lyman S, Koulouvaris P, Sherman S, et al. Epidemiology of anterior cruciate ligament reconstruction: trends, readmissions, and subsequent knee surgery. J Bone Joint Surg Am 2009;91:2321-8.

38 Thaunat M, Clowez G, Saithna A, et al. Reoperation rates after combined anterior cruciate ligament and anterolateral ligament reconstruction: a series of 548 patients from the SANTI Study Group with a minimum follow-up of 2 years. Am I Sports Med 2017:45:2569-77.

39 Spencer L, Burkhart TA, Tran MN, et al. Biomechanical analysis of simulated clinical testing and reconstruction of the anterolateral ligament of the knee. Am J Sports Med 2015;43:2189-97.

40 Blache Y, Dumas R, de Guise J, et al. Technical considerations in lateral extra-articular reconstruction coupled with anterior cruciate ligament reconstruction: a simulation study evaluating the influence of surgical parameters on control of knee stability. Clin Biomech 2019;61:136-43.

41 Wytrykowski K, Swider P, Reina N, et al. Cadaveric study comparing the biomechanical properties of grafts used for knee anterolateral ligament reconstruction. Arthroscopy 2016;32:2288-94.

42 Amis AA. Anterolateral knee biomechanics. Knee Surg Sports Traumatol Arthrosc 2017;25:1015-23.

43 Saithna A, Daggett M, Helito CP, et al. Clinical results of combined $A C L$ and anterolateral ligament reconstruction: a narrative review from the SANTI Study Group. J Knee Surg 2020. doi:10.1055/s-0040-1701220. [Epub ahead of print: 05 Feb 2020].

44 Ra HJ, Kim J-H, Lee D-H. Comparative clinical outcomes of anterolateral ligament reconstruction versus lateral extra-articular tenodesis in combination with anterior cruciate ligament reconstruction: systematic review and meta-analysis. Arch Orthop Trauma Surg 2020;140:923-31.

45 Sonnery-Cottet B, Daggett M, Fayard J-M, et al. Anterolateral Ligament Expert Group consensus paper on the management of internal rotation and instability of the anterior cruciate ligament - deficient knee. J Orthop Traumatol 2017:18:91-106. 